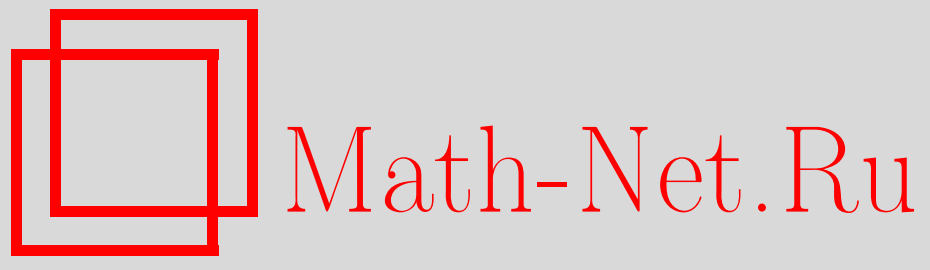

B. H. Jasiulis-Goldyn, J. K. Misiewicz, Weak Lévy-Khintchine representation for weak infinite divisibility, Теория вероятн. и ее примен., 2015, том 60, выпуск 1, 131-149

DOI: https://doi.org/10.4213/tvp4608

Использование Общероссийского математического портала Math-Net.Ru подразумевает, что вы прочитали и согласны с пользовательским соглашением http://www . mathnet.ru/rus/agreement

Параметры загрузки:

IP : 54.166 .219 .16

26 апреля 2023 г., 06:19:57

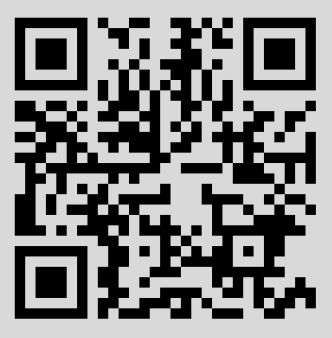


(C) 2015 г. JASIULIS-GOEDYN B. H. * MISIEWICZ J. K. **

\section{WEAK LÉVY-KHINTCHINE REPRESENTATION FOR WEAK INFINITE DIVISIBILITY}

Случайный вектор $\mathbf{X}$ является слабо устойчивым тогда и только тогда, когда для всех $a, b \in \mathbb{R}$ существует такая случайная величина $\Theta$, что $a \mathbf{X}+b \mathbf{X}^{\prime} \stackrel{d}{=} \mathbf{X} \Theta$, где $\mathbf{X}^{\prime}-$ независимая копия $\mathbf{X}$ и $\Theta$ не зависит от $\mathbf{X}$. Это равносильно (см. [12]) условию, что для всех $Q_{1}, Q_{2}$ существует такая случайная величина $\Theta$, что

$$
\mathbf{X} Q_{1}+\mathbf{X}^{\prime} Q_{2} \stackrel{d}{=} \mathbf{X} \Theta
$$

где $\mathbf{X}, \mathbf{X}^{\prime}, Q_{1}, Q_{2}, \Theta$ независимы и $\stackrel{d}{=}$ означает равенство распределений. Мы определим слабую обобщенную свертку мер формулой

$$
\mathscr{L}\left(Q_{1}\right) \otimes_{\mu} \mathscr{L}\left(Q_{2}\right)=\mathscr{L}(\Theta)
$$

если уравнение $(*)$ верно для $\mathbf{X}, Q_{1}, Q_{2}, \Theta$ и $\mu=\mathscr{L}(\mathbf{X})$. В статье изучены основные свойства этой свертки и распределений, которые бесконечно делимы относительно этой свертки. Основной результат этой работы является аналогом представления Леви-Хинчина $\otimes_{\mu}$-бесконечно делимых распределений.

Ключевые слова и фразы: слабо устойчивое распределение, обобщенная и слабая обобщенная свертка, бесконечная делимость, бесконечная делимость относительно обобщенной свертки.

1. Introduction. This paper contains the first step in construction Lévy processes in the sense of weak generalized convolution, i.e., the full characterization of infinitely divisible distributions in the sense of weak generalized convolution. The main example of such processes is the Bessel process, which is well known and widely used in stochastic modeling real processes (for definition an preliminary properties see [7]). The construction of the Bessel process is based on infinite divisibility of $\left\|\mathbf{W}_{t}\right\|_{2},\|\mathbf{x}\|_{2}^{2}=\sum x_{k}^{2}$,

${ }^{*}$ Institute of Mathematics, University of Wrocław, Pl. Grunwaldzki 2/4, 50-384 Wrocław, Poland; e-mail: jasiulis@math.uni.wroc.pl

** Faculty of Mathematics and Information Science, Warsaw University of Technology, Pl. Politechniki 1, 00-661 Warsaw, Poland; e-mail: j.misiewicz@mini.pw.edu.pl

1) This paper was partially supported by the Polish Government MNiSW grant № N201 371536 . 
where $\left\{\mathbf{W}_{t}\right\}$ is $n$-dimensional Brownian motion, with respect to the generalized weak convolution defined by the weakly stable uniform distribution $\omega_{n}$ on the unit sphere $S_{n-1} \subset \mathbb{R}^{n}$.

The infinite divisibility with respect to generalized convolution was studied by K. Urbanik in [21] and the corresponding Lévy-Khintchine formula is already known in this case. The weak generalized convolution does not coincide with generalized convolution considered by Urbanik. Infinite divisibility with respect to weak generalized convolutions described in this paper gives some additional properties of spectral representation and the corresponding spectral measure, which are useful in further constructions.

The idea of generalized convolution was introduced by K. Urbanik (see [20]) for probability measures on the positive half-line $[0, \infty)$. The basic properties of the generalized convolution together with infinite divisibility and stability of measures with respect to the generalized convolution were studied, e.g., in the papers [20]-[24] and still the problems and constructions based on generalized convolutions are of interest for many mathematicians, see, e.g., [25], [27].

In seventies of the last century J. Kucharczak and K. Urbanik (see, e.g., [8], [9], [19]) defined and studied weakly stable distributions on $[0, \infty)$, i.e., distributions $\mu$ on $[0, \infty)$ with the following property:

$$
\forall \quad a, b>0 \exists \lambda \text { on }[0, \infty) \quad T_{a} \mu * T_{b} \mu=\mu \circ \lambda,
$$

where $T_{0} \mu=\delta_{0},\left(T_{a} \mu\right)(A)=\mu(A / a)$ and $(\mu \circ \lambda)(A)=\int \mu(A / s) \lambda(d s)$ for every Borel set $A$ in $[0, \infty)$. Nowadays measures $\mu$ for which this condition holds are called $\mathbb{R}_{+}$-weakly stable. The $\mathbb{R}_{+}$-weakly stable distributions appeared as a result of noticing that most of generalized convolutions can be defined by some probability measure $\mu$ by the formula (1) (here $\lambda$ states for $\delta_{a}$ convoluted in generalized sense with $\delta_{b}$ ). For more information see, e.g., [9], [25]-[27]).

The paper [12] of J. Misiewicz, K. Oleszkiewicz, and K. Urbanik originated rather from theory of pseudo-isotropic distributions than from theory of generalized convolutions. It contains definition and basic properties of weakly stable distributions on $\mathbb{R}^{n}$, or on a separable Banach spaces $\mathbb{E}$. Precisely, a measure $\mu$ on $\mathbb{E}$ is weakly stable if

$$
\forall a, b \in \mathbb{R} \quad \exists \lambda \text { on } \mathbb{R} \quad T_{a} \mu * T_{b} \mu=\mu \circ \lambda .
$$

The slight change: $a, b>0$ for $\mathbb{R}_{+}$-weak stability to $a, b \in \mathbb{R}$ for weak stability turned out to make large difference. The authors proved that the condition (2) is equivalent to the following condition:

$$
\forall \lambda_{1}, \lambda_{2} \in \mathscr{P} \quad \exists \lambda \in \mathscr{P} \quad\left(\mu \circ \lambda_{1}\right) *\left(\mu \circ \lambda_{2}\right)=\mu \circ \lambda .
$$

If $\mu$ is not symmetric, then the measure $\lambda$ is uniquely determined, but if $\mu$ is symmetric, then only a symmetrization of $\lambda$ is uniquely determined. 
Moreover, we know that (see [12, Theorem 6]) if $\mu$ is weakly stable probability measure on a separable Banach space $\mathbb{E}$, then either there exists $a \in \mathbb{E}$ such that $\mu=\delta_{a}$, or there exists $a \in \mathbb{E} \backslash\{0\}$ such that $\mu=\left(\delta_{a}+\delta_{-a}\right) / 2$, or $\mu(\{a\})=0$ for every $a \in \mathbb{E}$. In this paper we assume that considered weakly stable measure is nontrivial, i.e., does not contain any atom.

Many interesting classes of weakly stable distributions are already known in the literature: symmetric Gaussian, symmetric stable, uniform distributions on the unit spheres $S^{n-1} \subset \mathbb{R}^{n}$, their $k$-dimensional projections and their deformations by linear operators. The extreme points in the set of $\ell_{1}$-symmetric distributions in $\mathbb{R}^{n}$ given by $\mathrm{S}$. Cambanis, R. Keener, and G. Simons (see [1]) are weakly stable. Strictly stable vectors are $\mathbb{R}_{+}$-weakly stable and it is still an open question whether or not they are weakly stable.

2. Preliminaries. In what follows we need some rather simple technical result (Lemma 1) concerning measure theory. The analog of this result for measures supported in $[0, \infty)$ was given by Urbanik (see [23], [24]).

Let $\mathscr{P}$ denote the set of all probability measures on the real line $\mathbb{R}$, $\mathscr{P}_{+}$- on the positive half-line $[0, \infty)$. Consider the one-point compactification of the real line $\overline{\mathbb{R}}$ with the compactifying point $\infty$ and let $\overline{\mathscr{P}}$ denote the set of probability measures on $\overline{\mathbb{R}}$. By $\mathscr{P}^{\infty}=\overline{\mathscr{P}} \backslash \mathscr{P}$ we denote the set of probability measures on $\overline{\mathbb{R}}$ with positive mass at $\infty$. For every $\mu \in \overline{\mathscr{P}}$ there exist uniquely determined measure $\mu^{\prime} \in \mathscr{P}$ and $p \in[0,1]$ such that $\mu=p \mu^{\prime}+(1-p) \delta_{\infty}$. The rescaling operator $T_{c}: \mathscr{P} \rightarrow \mathscr{P}, c \in \mathbb{R} \backslash\{0\}$, has the natural extension to the set $\overline{\mathscr{P}}$ :

$$
T_{c}\left(p \mu^{\prime}+(1-p) \delta_{\infty}\right)=p T_{c} \mu^{\prime}+(1-p) \delta_{\infty}
$$

If the sequence of measures $\mu_{n}$ converges weakly to $\mu$ we will write $\mu_{n} \rightarrow \mu$.

Proposition 1. Assume that $\mu_{n}, \mu \in \overline{\mathscr{P}}, \mu \neq \delta_{0}, \mu_{n} \rightarrow \mu$ and let $c_{n} \rightarrow \infty$. Then every accumulation point of the sequence $T_{c_{n}} \mu_{n}$ belongs to $\mathscr{P} \infty$.

P r o o f. Let $\mu=a \delta_{0}+(1-a) \mu_{1}$, where $\mu_{1}(\{0\})=0$ and $a \in[0,1)$. For every $\delta<1-a$ let $(-\varepsilon, \varepsilon)$ be a continuity set for the measure $\mu$ such that $\mu((-\varepsilon, \varepsilon))<a+\delta$. Then for every $A=(-m, m)^{c}$ and $n$ large enough we have

$$
T_{c_{n}} \mu_{n}(A)=\mu_{n}\left(A / c_{n}\right)>\mu_{n}\left((-\varepsilon, \varepsilon)^{c}\right) \rightarrow \mu\left((-\varepsilon, \varepsilon)^{c}\right)>1-a-\delta .
$$

Since $m$ can be taken arbitrarily large and $\delta$ can be taken arbitrarily small, this shows that every accumulation point of $T_{c_{n}} \mu_{n}$ has the atom at $\infty$ of the weight $1-a$.

Lemma 1. Assume that $\mu_{n}, \mu \in \mathscr{P}, \mu \neq \delta_{0}, T_{a_{n}} \mu_{n} \rightarrow \mu$ and assume that $T_{b_{n}} \mu_{n}$ is conditionally compact in $\mathscr{P}$. Then the sequence $\left(b_{n} / a_{n}\right)$ is bounded and the set of accumulation points of the sequence $T_{b_{n}} \mu_{n}$ coincides 
with the set of measures $T_{c} \mu$, where $c$ belongs to the set of accumulation points of the sequence $\left(b_{n} / a_{n}\right)$.

P r o o f. Assume that $T_{b_{n_{k}}} \mu_{n_{k}} \rightarrow \nu \in \mathscr{P}$. If $d_{k}=b_{n_{k}} / a_{n_{k}} \rightarrow \infty$, then

$$
T_{d_{k}} T_{a_{n_{k}}} \mu_{n_{k}}=T_{b_{n_{k}}} \mu_{n_{k}} \rightarrow \nu
$$

thus, by Proposition $1, \nu \in \mathscr{P}^{\infty}$ in contradiction with our assumption. Without loss of generality we can assume now that $d_{k} \rightarrow c$ for some $c \in \mathbb{R}$. Then $T_{b_{n_{k}}} \mu_{n_{k}} \rightarrow \nu$ and

$$
T_{b_{n_{k}}} \mu_{n_{k}}=T_{d_{k}}\left(T_{a_{n_{k}}} \mu_{n_{k}}\right) \rightarrow T_{c} \mu,
$$

thus $\nu=T_{c} \mu$, which was to be proved.

3. Generalized weak convolution. In this section we define and study properties of weak generalized convolution. We define also distributions infinitely divisible with respect to weak generalized convolution and prove the semigroup structure properties of theirs powers.

For a measure $\mu \in \mathscr{P}(\mathbb{E})$ we define $\mathscr{M}(\mu)=\{\mu \circ \lambda: \lambda \in \mathscr{P}\}$, the set of all scale mixtures of the measure $\mu$. For a symmetric random vector $\mathbf{X}$ independent of random variable $\Theta$ we have $\mathbf{X} \Theta \stackrel{d}{=} \mathbf{X}|\Theta|$. This implies that if $\mu$ is a symmetric probability distribution, then for every $\lambda \in \mathscr{P}$

$$
\mu \circ \lambda=\mu \circ|\lambda|,
$$

where $|\lambda|$ is the distribution of $|\theta|$ if $\lambda$ is the distribution of $\theta$.

$\mathrm{D}$ e f i n i t i o n 1 . Let $\mu \in \mathscr{P}(\mathbb{E})$ be a nontrivial weakly stable measure, and let $\lambda_{1}, \lambda_{2}$ be probability measures on $\mathbb{R}$. If

$$
\left(\mu \circ \lambda_{1}\right) *\left(\mu \circ \lambda_{2}\right)=\mu \circ \lambda,
$$

then the generalized convolution of the measures $\lambda_{1}, \lambda_{2}$ with respect to the measure $\mu$ (notation $\lambda_{1} \otimes_{\mu} \lambda_{2}$ ) is defined as follows

$$
\lambda_{1} \otimes_{\mu} \lambda_{2}= \begin{cases}\lambda & \text { if } \mu \text { is not symmetric; } \\ |\lambda| & \text { if } \mu \text { is symmetric. }\end{cases}
$$

We separated symmetric and nonsymmetric cases in the formula above because (see [12]) if $\mu$ is symmetric, then the measure $|\lambda|$ (not $\lambda$ itself) is uniquely determined. Sometimes, if this is more adequate for the real process, the definition of weak generalized convolution of measures $\lambda_{1}, \lambda_{2}$ for symmetric weakly stable measure $\mu$ can be formulated as $\lambda_{1} \otimes_{\mu} \lambda_{2}=$ $\left(|\lambda|+T_{-1}|\lambda|\right) / 2$. This is possible because the symmetric mixing measure is also uniquely determined by its mixture with a weakly stable measure $\mu$.

The proof of the following lemma is standard and will be omitted. 
Lemma 2. If the weakly stable measure $\mu \in \mathscr{P}(\mathbb{E})$ is not trivial, then

1) $\lambda_{1} \otimes_{\mu} \lambda_{2}$ is uniquely determined;

2) $\lambda_{1} \otimes_{\mu} \lambda_{2}=\lambda_{2} \otimes_{\mu} \lambda_{1}$

3) $\left(\lambda_{1} \otimes_{\mu} \lambda_{2}\right) \otimes_{\mu} \lambda_{3}=\lambda_{1} \otimes_{\mu}\left(\lambda_{2} \otimes_{\mu} \lambda_{3}\right)$;

4) $\lambda \otimes_{\mu} \delta_{0}=\lambda\left(\lambda \otimes_{\mu} \delta_{0}=|\lambda|\right.$ if $\mu$ is symmetric $)$;

5) $\left(p \lambda_{1}+(1-p) \lambda_{2}\right) \otimes_{\mu} \lambda=p\left(\lambda_{1} \otimes_{\mu} \lambda\right)+(1-p)\left(\lambda_{2} \otimes_{\mu} \lambda\right)$ for each $p \in[0,1]$;

6) $T_{a}\left(\lambda_{1} \otimes_{\mu} \lambda_{2}\right)=\left(T_{a} \lambda_{1}\right) \otimes_{\mu}\left(T_{a} \lambda_{2}\right)$.

In $1964 \mathrm{~K}$. Urbanik introduced the definition of a generalized convolution $\diamond$ as a binary operation $\diamond$ on the space $\mathscr{P}_{+}^{2}$ having the properties 1) -6 ) with $\otimes$ replaced by $\diamond$ and such that additionally the following condition holds:

(v) there exists a sequence of positive numbers $\left(c_{n}\right)$ such that $T_{c_{n}} \delta_{1}^{\diamond n}$ converges weakly to a measure $\nu \neq \delta_{0}$ (here $\lambda^{\diamond n}=\lambda \diamond \cdots \diamond \lambda$ denotes the generalized convolution of $n$ identical measures $\lambda$ ).

Notice that for the weak generalized convolution the property (v) does not have to hold. In [4] we can find a wide discussion of the properties of generalized convolutions without (v) assumption defined for measures on the whole real line. However it was shown in [12] that if the measure $\mu$ has a finite $\varepsilon$ moment for some $\varepsilon>0$, then there exists a measure $\lambda$ such that $\lambda \circ \mu$ is symmetric $\alpha$-stable for some (and then for every) $\alpha \leqslant \min \{\varepsilon, 2\}$. This means that $T_{c_{n}} \lambda^{\otimes n}=\lambda$ for properly chosen sequence $\left(c_{n}\right)$ and the property $(\mathrm{v})$ holds if we replace $\delta_{1}$ by $\lambda$.

$\mathrm{Ex} \mathrm{a} \mathrm{m} \mathrm{ple} \mathrm{1.} \mathrm{It} \mathrm{is} \mathrm{known} \mathrm{(see} \mathrm{[7])} \mathrm{that} \mathrm{the} \mathrm{random} \mathrm{vector} \mathbf{U}^{n}=$ $\left(U_{1}, \ldots, U_{n}\right)$ with the uniform distribution $\omega_{n}$ on the unit sphere $S_{n-1} \subset \mathbb{R}^{n}$ is weakly stable but the easiest way to see this is using properties of rotationally invariant vectors.

Let us recall that the random vector $\mathbf{X} \in \mathbb{R}^{n}$ is rotationally invariant (sometimes the name spherically symmetric is used) if $L(\mathbf{X}) \stackrel{d}{=} \mathbf{X}$ for every unitary linear operator $L: \mathbb{R}^{n} \rightarrow \mathbb{R}^{n}$. It is known (see [18] for the details) that the following conditions are equivalent:

(a) $\mathbf{X} \in \mathbb{R}^{n}$ is rotationally invariant;

(b) $\mathbf{X} \stackrel{d}{=} \Theta \mathbf{U}^{n}$, where $\Theta:=\|\mathbf{X}\|_{2}$ is independent of $\mathbf{U}:=\mathbf{X} /\|\mathbf{X}\| \stackrel{d}{=} \mathbf{U}^{n}$;

(c) the characteristic function of $\mathbf{X}$ has the form

$$
\mathbf{E} e^{i<\xi, \mathbf{X}>}=\varphi \mathbf{X}(\xi)=\varphi\left(\|\xi\|_{2}\right)
$$

for some symmetric function $\varphi: \mathbb{R} \rightarrow \mathbb{R}$.

Now let $\mathscr{L}\left(\Theta_{1}\right)=\lambda_{1}, \mathscr{L}\left(\Theta_{2}\right)=\lambda_{2}$ be such that $\Theta_{1}, \Theta_{2}, \mathbf{U}^{n 1}, \mathbf{U}^{n 2}$ are independent, $\mathbf{U}^{n 1} \stackrel{d}{=} \mathbf{U}^{n 2} \stackrel{d}{=} \mathbf{U}^{n}$. Consider the characteristic function $\psi$ of the vector $\mathbf{Z}=\Theta_{1} \mathbf{U}^{n 1}+\Theta_{2} \mathbf{U}^{n 2}$

$$
\begin{aligned}
\psi(\xi) & =\mathbf{E} \exp \left\{i\left\langle\xi, \Theta_{1} \mathbf{U}^{n 1}+\Theta_{2} \mathbf{U}^{n 2}\right\rangle\right\} \\
& =\iint \varphi_{1}\left(\|\xi\|_{2}|s|\right) \varphi_{2}\left(\|\xi\|_{2}|t|\right) \lambda_{1}(d s) \lambda_{2}(d t) .
\end{aligned}
$$


We see that $\psi$ also depends only on $\|\xi\|_{2}$ thus the condition (c) is satisfied and $\mathbf{Z}$ is rotationally invariant. By condition (b) we have that $\mathbf{Z} \stackrel{d}{=} \Theta \mathbf{U}^{n}$ for the random variable $\Theta \stackrel{d}{=}\|\mathbf{Z}\|_{2}$, which we denote by $\Theta_{1} \oplus_{\omega_{n}} \Theta_{2}$. This means that $\mathbf{U}^{n}$ is weakly stable and the weak generalized convolution $\otimes_{\omega_{n}}$ defines in a natural way a weak generalized summation by the following formula

$$
\Theta_{1} \oplus_{\omega_{n}} \Theta_{2}=\left\|\Theta_{1} \mathbf{U}^{n 1}+\Theta_{2} \mathbf{U}^{n 2}\right\|_{2}
$$

D e f i n i t i o n 2. Let $\mathscr{L}(\Theta)=\lambda$ and let $\mu=\mathscr{L}(\mathbf{X})$ be a weakly stable measure on $\mathbb{E}$. We say that the measure $\lambda$ (and the corresponding variable $\Theta$ ) is $\mu$-weakly infinitely divisible if for every $n \in \mathbb{N}$ there exists a probability measure $\lambda_{n}$ such that

$$
\lambda=\lambda_{n}^{\otimes n} \equiv \lambda_{n}^{n} \stackrel{\text { def }}{=} \lambda_{n} \otimes_{\mu} \cdots \otimes_{\mu} \lambda_{n}, \quad(n \text {-times })
$$

where (for the uniqueness) $\lambda_{n} \in \mathscr{P}_{+}$if $\mu$ is $\mathbb{R}_{+}$-weakly stable or if $\mu$ is symmetric, and $\lambda_{n} \in \mathscr{P}$ if $\mu$ is weakly stable nonsymmetric.

Notice that if $\lambda$ is $\mu$-weakly infinitely divisible then the measure $\mu \circ \lambda$ is infinitely divisible in the usual sense. However, as it is shown in the following example, infinite divisibility of $\mu \circ \lambda$ does not have to imply $\mu$ infinite divisibility of $\lambda$.

$\mathrm{Ex} \mathrm{a} \mathrm{m} \mathrm{p} \mathrm{l} \mathrm{e} \mathrm{2.} \mathrm{It} \mathrm{is} \mathrm{easy} \mathrm{to} \mathrm{see} \mathrm{that} \mathrm{the} \mathrm{symmetric} \alpha$-stable distribution $\gamma_{\alpha}$ on $\mathbb{R}$ is weakly stable and the weak generalized convolution naturally defines weak generalized summation in the following way:

$$
\Theta_{1} \oplus_{\gamma_{\alpha}} \Theta_{2} \stackrel{d}{=}\left(\left|\Theta_{1}\right|^{\alpha}+\left|\Theta_{2}\right|^{\alpha}\right)^{1 / \alpha}
$$

for independent random variables $\Theta_{1}, \Theta_{2}$. This implies that $\Theta$ is $\otimes_{\gamma_{\alpha}}{ }^{-}$ infinitely divisible if and only if $|\Theta|^{\alpha}$ is infinitely divisible in the classical sense. On the other hand it is known (see [6], [11]) that for $\alpha \leqslant 1$ and every $\lambda \in \mathscr{P}$ the measure $\gamma_{\alpha} \circ \lambda$ is infinitely divisible in the usual sense, thus it is infinitely divisible even in the case when $|\Theta|^{\alpha}$ is not infinitely divisible.

The following lemma was proven in [14].

Lemma 3. Let $\mu$ be a weakly stable distribution, $\mu \neq \delta_{0}$. If $\lambda$ is $\mu$ weakly infinitely divisible, then there exists a family $\left\{\lambda^{r}: r \geqslant 0\right\}$ such that

1) $\lambda^{0}=\delta_{0}, \lambda^{1}=\lambda$

2) $\lambda^{r} \otimes_{\mu} \lambda^{s}=\lambda^{r+s}, r, s \geqslant 0$

3) $\lambda^{r} \rightarrow \delta_{0}$ if $r \rightarrow 0$.

4. $\mu$-weak compound Poisson measures. Let us start from the following definition.

$\mathrm{D}$ e f i n i t i o n 3 . Let $\mu$ be a nontrivial weakly stable probability measure on a separable Banach space $\mathbb{E}$. For every $\lambda \in \mathscr{P}$ and every $a>0$ 
the $\mu$-weak compound Poisson measure is defined by the following formula:

$$
\operatorname{Exp}_{\otimes_{\mu}}(a \lambda) \stackrel{\text { def }}{=} e^{-a} \sum_{k=0}^{\infty} \frac{a^{k}}{k !} \lambda^{\otimes k} .
$$

Proposition 2. For every $\lambda \in \mathscr{P}$, every $a>0$, and every nontrivial weakly stable probability measure on $\mathbb{E}$ the $\mu$-weak compound Poisson measure is a well defined $\mu$-weakly infinitely divisible probability measure. Moreover,

$$
\mu \circ \operatorname{Exp}_{\otimes_{\mu}}(a \lambda)=\exp (a(\mu \circ \lambda)) .
$$

P r o o f. Let $a(n)=\sum_{k=0}^{n} a^{k} / k$ !, and with the notation $\lambda^{k}=\lambda^{\otimes_{\mu} k}$, let

$$
\mathscr{A}=\left\{\nu_{n}=\frac{1}{a(n)} \sum_{k=0}^{n} \frac{a^{k}}{k !} \lambda^{k}: n \in \mathbb{N}\right\}
$$

and

$$
\mathscr{C}=\left\{\mu \circ \nu_{n}: n \in \mathbb{N}\right\}=\left\{\frac{1}{a(n)} \sum_{k=0}^{n} \frac{a^{k}}{k !}(\mu \circ \lambda)^{* k}: n \in \mathbb{N}\right\}
$$

Since

$$
\mu \circ \nu_{n} \rightarrow e^{-c} \sum_{k=0}^{\infty} \frac{a^{k}}{k !}(\mu \circ \lambda)^{* k}=\exp (a \mu \circ \lambda),
$$

the family $\mathscr{C}$ is tight. Lemma 2 in [12] implies that the family $\mathscr{A}$ is also tight and there exists a measure $\nu$ such that $\nu_{n_{k}} \Rightarrow \nu$ for some subsequence $\left(\nu_{n_{k}}\right) \subset \mathscr{A}$. Of course $\mu \circ \nu_{n_{k}} \Rightarrow \mu \circ \nu$, and $\lim \mu \circ \nu_{n_{k}}=\lim \mu \circ \nu_{n}=\exp (c \mu \circ \lambda)$, we conclude that

$$
\mu \circ \nu=\exp (a \mu \circ \lambda)
$$

Uniqueness of the measure $\nu$ follows from the weak stability of $\mu$ and from the fact that weakly stable distributions are reducible. Thus there is only one accumulation point $\nu$ for the family $\mathscr{A}$ and we will denote it by

$$
\operatorname{Exp}_{\otimes_{\mu}}(a \lambda) \stackrel{\text { def }}{=} \nu=e^{-a} \sum_{k=0}^{\infty} \frac{a^{k}}{k !} \lambda^{\otimes k}
$$

To see that $\operatorname{Exp}_{\otimes_{\mu}}(a \lambda)$ is $\mu$-weakly infinitely divisible it is enough to notice that

$$
\left[\left(\mu \circ \operatorname{Exp}_{\otimes_{\mu}}\left(\frac{a}{n} \lambda\right)\right)\right]^{* n}=\mu \circ \operatorname{Exp}_{\otimes_{\mu}}(a \lambda) .
$$

$\mathrm{Ex}$ a $\mathrm{m} \mathrm{p} \mathrm{l} \mathrm{e} 3$. Let $\mu_{\alpha}$ be the weakly stable distribution defining the Kendall weak generalized convolution, i.e., $\widehat{\mu_{\alpha}}(t)=\left(1-|t|^{\alpha}\right)_{+}$, where $0<\alpha \leqslant$ 1. Then the measure $\operatorname{Exp}\left(c \mu_{\alpha}\right)=\mu_{\alpha} \circ \operatorname{Exp}_{\otimes_{\mu_{\alpha}}}\left(c \delta_{1}\right)$ has the characteristic function

$$
\exp \left\{-c\left(1-\widehat{\mu_{\alpha}}(t)\right)\right\}=\exp \left\{-c\left(|t|^{\alpha} \wedge 1\right)\right\}
$$


In order to describe the measure $\operatorname{Exp}_{\otimes_{\mu_{\alpha}}}\left(c \delta_{1}\right)$ put $x^{-1}=|t|^{\alpha}$ and $G(u)=$ $\lambda\left\{s: s^{\alpha}<u\right\}$. Then

$$
\exp \left\{-c\left(x^{-1} \wedge 1\right)\right\}=\int_{0}^{\infty}\left(1-x^{-1} u\right)_{+} d G(u)=x^{-1} \int_{0}^{x} G(u) d u .
$$

For $x \in(0,1)$ we obtain that $e^{-c}=x^{-1} \int_{0}^{x} G(u) d u$ which implies that $G(x)=$ $e^{-c}$. For $x>1$ the equality

$$
\int_{0}^{x} G(u) d u=x \exp \left\{-c x^{-1}\right\}
$$

implies that $G(x)=\left(1+c x^{-1}\right) e^{-c x^{-1}}$. Now it is easy to see that

$$
\operatorname{Exp}_{\otimes_{\mu_{\alpha}}}\left(c \delta_{1}\right)(d u)=e^{-c} \delta_{0}(d u)+c e^{-c} \delta_{1}(d u)+\frac{c^{2} \alpha}{u^{2 \alpha+1}} e^{-c u^{-\alpha}} \mathbf{1}_{(1, \infty)}(u) d u .
$$

5. $\mu$-weakly stable distributions. From now on we will assume that $\mathbb{E}=\mathbb{R}$. This will not cause loss of generality since for any weakly stable measure $\mu \in \mathscr{P}(\mathbb{E})$ nondegenerated to a subspace and any its one-dimensional projection $\mu_{1} \neq \delta_{0}$ of the measure $\mu$ we have

$$
\otimes_{\mu}=\otimes_{\mu_{1}} .
$$

A measure $\lambda \in \mathscr{P} \backslash\left\{\delta_{0}\right\}$ is $\mu$-weakly stable with respect to a nontrivial weakly stable measure $\mu$ if there exists a sequence of positive numbers $\left(c_{n}\right)_{n}$ and a measure $\nu \in \mathscr{P}$ such that $T_{c_{n}} \nu^{n} \rightarrow \lambda$. The set of all $\mu$-weakly stable measures we denote by $\mathscr{S}(\mu)$. Let

$$
\mathscr{S}_{p}(\mu)=\left\{\lambda \in \mathscr{P} \backslash\left\{\delta_{0}\right\}: T_{a} \lambda \otimes_{\mu} T_{b} \lambda=T_{g_{p}(a, b)} \lambda\right\},
$$

where $g_{p}(a, b)=\left(|a|^{p}+|b|^{p}\right)^{1 / p}$

$\mathrm{R} \mathrm{e} \mathrm{mark} 1$. If $\lambda \in \mathscr{S}_{p}(\mu)$, then $T_{n^{-1 / p}} \lambda^{n}=\lambda$. This shows that $\mathscr{S}_{p}(\mu) \subset \mathscr{S}(\mu)$ for every $p>0$.

$\mathrm{R}$ e $\mathrm{m}$ a r k 2. For every nontrivial weakly stable measure $\mu$ and every $p>2$ we have $\mathscr{S}_{p}(\mu)=\varnothing$.

To see this assume that $\lambda \in \mathscr{S}_{p}(\mu)$. Since $T_{a} \lambda \otimes_{\mu} T_{b} \lambda=T_{g_{p}(a, b)} \lambda$ for all $a, b>0$ we get $T_{a} \lambda \circ \mu * T_{b} \lambda \circ \mu=T_{g_{p}(a, b)} \lambda \circ \mu$ for all $a, b>0$. This means that the measure $\lambda \circ \mu$ is strictly stable with the index of stability $p$. The general theory of stable distributions states that $p \leqslant 2$. In particular, we have that $S_{p}(\mu) \neq \varnothing$ if and only if $\mathscr{M}(\mu)$ contains symmetric $p$-stable distributions.

$\mathrm{R}$ e mark 3 . If $\mu$ is a nontrivial weakly stable measure and $\mathscr{S}_{p}(\mu) \neq \varnothing$ for some $p \in(0,2]$, then $\mathscr{S}_{q}(\mu) \neq \varnothing$ for all $q \in(0, p]$.

Let $\lambda \in \mathscr{S}_{p}(\mu)$. Then the random variable $X$ with distribution $\lambda \circ \mu$ is strictly stable with the index of stability $p$. For $r=q / p, q \in(0, p)$, let $\gamma_{r}^{+}$ denotes the $r$-stable distribution concentrated on the positive half-line with 
the Laplace transform $e^{-t^{r}}$. Let $\Theta$ be the random variable with distribution $\gamma_{r}^{+}$independent of $X$. It is known that the random variable $Y=X \Theta^{1 / p}$ is strictly stable with index of stability $q$. Denoting by $\gamma_{r, p}^{+}$the distribution of $\Theta^{1 / p}$ we can write that

$$
\mathscr{L}(Y)=(\lambda \circ \mu) \circ \gamma_{r, p}^{+}=\left(\lambda \circ \gamma_{r, p}^{+}\right) \circ \mu,
$$

which shows that $\lambda \circ \gamma_{r, p}^{+} \in \mathscr{S}_{q}(\mu)$.

$\mathrm{R}$ e $\mathrm{m}$ a r $\mathrm{k}$ 4. If $\mu$ is a symmetric nontrivial weakly stable measure and $\mathscr{S}_{p}(\mu) \neq \varnothing$, then $p \in(0,2]$ and there exists $\lambda_{p}$ such that $\lambda_{p} \circ \mu=\gamma_{p}$, where $\gamma_{p}$ is the standard symmetric $p$-stable distribution with the characteristic function $\exp \left\{-|t|^{p}\right\}$ and

$$
\mathscr{S}_{p}(\mu)=\left\{T_{a} \lambda_{p}: a>0\right\} .
$$

The first statement follows from the fact that $e^{-|t|^{\alpha}}$ is a characteristic function if and only if $p \in(0,2]$. To see the second assume that $\lambda, \nu \in \mathscr{S}_{p}(\mu)$. By the argument used in Remark 2 we know that $\lambda \circ \mu$ and $\nu \circ \mu$ are symmetric $p$-stable distributions and there exist positive numbers $a, b$ such that $\lambda \circ \mu=T_{a} \gamma_{p}$ and $\nu \circ \mu=T_{b} \gamma_{p}$. Since the measure $\mu$ is reducible $T_{a^{-1}} \lambda=T_{b^{-1}} \nu:=\lambda_{p}$, which was to be shown.

Proposition 3. If $\mu$ is a nontrivial symmetric weakly stable measure, then

$$
\mathscr{S}(\mu)=\bigcup_{p \in(0,2]} \mathscr{S}_{p}(\mu) .
$$

P r o o f. Let $\lambda \in \mathscr{S}(\mu)$. This means that there exists a sequence of positive numbers $\left(c_{n}\right)_{n}$ and a probability measure $\nu$ such that $T_{c_{n}} \nu^{n} \rightarrow \lambda$. Lemma 2.7 in [4] states that $c_{n} \rightarrow 0$ and $c_{n} / c_{n+1} \rightarrow 1$ if $n \rightarrow \infty$. This implies that for every pair of positive numbers we can find a subsequences $\left(c_{n_{k}}\right)_{k}$ and $\left(c_{m_{k}}\right)_{k}$ satisfying the condition

$$
\lim _{k \rightarrow \infty} \frac{c_{n_{k}}}{c_{m_{k}}}=\frac{y}{x}
$$

For $b_{k}=x c_{n_{k}} / c_{m_{k}}, d_{k}=x c_{n_{k}} / c_{n_{k}+m_{k}}$ we see that

$$
T_{d_{k}} T_{c_{n_{k}+m_{k}}} \nu^{n_{k}+m_{k}}=T_{x}\left(T_{c_{n_{k}}} \nu^{n_{k}}\right) \otimes_{\mu} T_{d_{k}}\left(T_{c_{n_{k}}} \nu^{n_{k}}\right) .
$$

The right-hand side of the above equality tends to $T_{x} \lambda \otimes_{\mu} T_{y} \lambda$. By Lemma 1 we obtain that the $\operatorname{limit} d=\lim _{n \rightarrow \infty} d_{k}$ exists. This shows that the lefthand side of the above equality converges to $T_{d} \lambda$ as $k \rightarrow \infty$. Consequently, $T_{x}(\mu \circ \lambda) * T_{y}(\mu \circ \lambda)=T_{d}(\mu \circ \lambda)$, which is the characterizing equation for stable distribution, thus $\lambda \circ \mu$ is $p$-stable for some $p \in(0,2]$ and $d=g_{p}(x, y)$.

The following theorem describes a characterizing parameter $\varkappa(\mu)$ for the weakly stable distribution $\mu$. This parameter plays a similar role as the parameter $\alpha$ for $\alpha$-stable distribution. 
Theorem 1. For every symmetric weakly stable distribution $\mu$

$$
\begin{aligned}
\varkappa=\varkappa(\mu) & :=\sup \left\{p \in[0,2]: \mathscr{S}_{p}(\mu) \neq \varnothing\right\} \\
& =\sup \left\{p \in[0,2]: \int_{\mathbb{R}}|x|^{p} \mu(d x)<\infty\right\} \\
& =\sup \left\{p \in[0,2]: \gamma_{p} \in \mathscr{M}(\mu)\right\},
\end{aligned}
$$

where, by our convention, the supremum over the empty set equals zero.

P r o o f. Let

$$
\begin{aligned}
& \varkappa_{1}:=\sup \left\{p \in[0,2]: \int_{\mathbb{R}}|x|^{p} \mu(d x)<\infty\right\}, \\
& \varkappa_{2}:=\sup \left\{p \in[0,2]: \gamma_{p} \in \mathscr{M}(\mu)\right\} .
\end{aligned}
$$

Notice first that $\gamma_{p} \in \mathscr{M}(\mu)$ if and only if there exists $\lambda_{p} \in \mathscr{P}$ such that $\mu \circ \lambda_{p}=\gamma_{p}$. Since $T_{a} \gamma_{p} * T_{b} \gamma_{p}=T_{g_{p}(a, b)} \gamma_{p}$ this is equivalent to $T_{a} \lambda_{p} \otimes_{\mu} T_{b} \lambda_{p}=$ $T_{g_{p}(a, b)} \lambda_{p}$ which means that $\mathscr{S}_{p}(\mu) \neq \varnothing$. This proves that $\varkappa=\varkappa_{2}$.

It was shown in [12, Theorem 5, Remark 3] that if $0<p<\varkappa_{1}$, then $\gamma_{p} \in \mathscr{M}(\mu)$. This means that $p \leqslant \varkappa_{2}$ and, consequently, $\varkappa_{1} \leqslant \varkappa_{2}$. On the other hand, if $0<p<\varkappa_{2}$ and $\mu \circ \lambda_{p}=\gamma_{p}$, then for every $0<\alpha<p$

$$
\infty>\int_{\mathbb{R}}|x|^{\alpha} \gamma_{p}(d x)=\int_{\mathbb{R}}|x|^{\alpha} \mu(d x) \int_{\mathbb{R}}|s|^{\alpha} \lambda_{p}(d s),
$$

which shows that $\alpha \leqslant \varkappa_{1}$ and, consequently, $\varkappa_{2} \leqslant \varkappa_{1}$.

Corollary 1. If $\mu$ is a symmetric, nontrivial weakly stable measure and $\varkappa(\mu)=2$, then for every $p>0$

$$
\int_{\mathbb{R}}|x|^{p} \mu(d x)<\infty
$$

P r o o f. We know that there exists $\lambda_{2}$ such that $\lambda_{2} \circ \mu$ is a symmetric Gaussian measure. Then for every $p>0$ we have

$$
\infty>\int_{\mathbb{R}}|x|^{p}\left(\mu \circ \lambda_{2}\right)(d x)=\int_{\mathbb{R}}|x|^{p} \mu(d x) \int_{\mathbb{R}}|s|^{p} \lambda_{2}(d s),
$$

which implies that both factors on the right-hand side of this formula are finite.

E x a m p l e 4. Let $\mu_{\alpha}, \alpha \in(0,1]$, be a weakly stable measure defining the weak Kendall generalized convolution (see [5]). The characteristic function of $\mu_{\alpha}$ is of the form $\widehat{\mu_{\alpha}}(t)=\left(1-|t|^{\alpha}\right)_{+}$. Since $\lim _{n \rightarrow \infty}\left(\widehat{\mu_{\alpha}}\left(t / n^{1 / \alpha}\right)\right)^{n}=$ $\exp \left\{-|t|^{\alpha}\right\}$, we see that $T_{n^{1 / \alpha}} \mu^{* n}$ converges weakly to the standard symmetric $\alpha$-stable measure $\gamma_{\alpha}$. The set $\mathscr{M}\left(\mu_{\alpha}\right)$ is closed under taking weak limits thus $\gamma_{\alpha}=\mu_{\alpha} \circ \nu$ for some $\nu \in \mathscr{P}$. It means that for every $p \leqslant \alpha$ there exists a probability measure $\nu_{p} \in \mathscr{P}_{+}$such that

$$
\exp \left\{-|t|^{p}\right\}=\int_{0}^{\infty}\left(1-|t s|^{\alpha}\right)+\nu_{p}(d s)
$$


Integrating by parts, with the notation $x=|t|^{-\alpha}$ and $F_{p}(u)=\nu_{p}\left\{s^{\alpha}<u\right\}$, we obtain

$$
\exp \left\{-x^{-p / \alpha}\right\}=\int_{0}^{x}\left(1-x^{-1} u\right)_{+} d F_{p}(u)=x^{-1} \int_{0}^{x} F_{p}(u) d u .
$$

Consequently,

$$
F_{p}(u)=\left(1+\frac{p}{\alpha} u^{-p / \alpha}\right) e^{-u^{-p / \alpha}}
$$

Now it is easy to see that the cumulative distribution function $G_{p}$ for the measure $\nu_{p}$ is of the form $G_{p}(s)=F_{p}\left(s^{\alpha}\right)$, so $\nu_{p}$ is absolutely continuous with respect to the Lebesgue measure with the density

$$
g_{p}(s)=\frac{p}{\alpha}\left((\alpha-p) s^{-p-1}+p s^{-(2 p+1)}\right) e^{-s^{-p}} \mathbf{1}_{(0, \infty)}(s) .
$$

The measure $\nu_{\alpha}$ with density $g_{\alpha}$ on $[0, \infty)$ is $\mu_{\alpha}$-weakly $\alpha$-stable. In particular, it means that $\varkappa\left(\mu_{\alpha}\right) \geqslant \alpha$. On the other hand we have that $1-\widehat{\mu_{\alpha}}(t)=|t|^{\alpha}$ in the neighborhood of zero, thus $\mu_{\alpha}\{|x|>r\} \sim r^{-\alpha}$ as $r \rightarrow \infty$ and

$$
\int|x|^{\alpha} \mu_{\alpha}(d x)=\infty
$$

which implies that $\alpha \leqslant \varkappa\left(\mu_{\alpha}\right)$. In a similar way for every $p<\alpha$ we can show that

$$
\begin{aligned}
\exp \left\{-|t|^{p}\right\} & =\exp \left\{-\int_{0}^{\infty}\left(1-\left(1-|t s|^{\alpha}\right)_{+}\right) \frac{p(\alpha-p)}{\alpha s^{p+1}} d s\right\} \\
& =\exp \left\{-\int_{0}^{\infty} \int_{\mathbb{R}}\left(1-e^{i t x s}\right) \mu_{\alpha}(d x) \frac{p(\alpha-p)}{\alpha s^{p+1}} d s\right\} .
\end{aligned}
$$

This means that the Lévy measure in the Lévy-Khintchine representation for symmetric $p$-stable measure with the characteristic function $\exp \left\{-|t|^{p}\right\}$ can be written as $\mu_{\alpha} \circ \lambda_{p}$, where $\lambda_{p}$ is concentrated on $(0, \infty)$ and has the density $p(\alpha-p) \alpha^{-1} s^{-p-1}$.

For $p=\alpha$ such measure $\lambda_{\alpha}$ does not exists, but we have

$$
\exp \left\{-|t|^{\alpha}\right\}=\lim _{p \nearrow \alpha} \exp \left\{-\int_{0}^{\infty}\left(1-\widehat{\mu_{\alpha}}(t s)\right) \frac{p(\alpha-p)}{\alpha s^{p+1}} d s\right\} .
$$

E x a m p l e 6. Consider the weakly stable Kingman distributions

$$
\omega_{s, 1}(d x)=\frac{\Gamma(s+1)}{\sqrt{\pi} \Gamma(s+1 / 2)}\left(1-x^{2}\right)^{s-1 / 2} \mathbf{1}_{(-1,1)}(x) d x,
$$

where $s>-1 / 2$. Since

$$
\int_{\mathbb{R}} x^{2} \omega_{s, 1}(d x)=\frac{1}{s+1}<\infty
$$


we see that $\varkappa\left(\omega_{s, 1}\right)=2$. In particular, this means that there exists a probability measure $\nu_{s, 2}$ such that $\omega_{s, 1} \circ \nu_{s, 2}$ is the Gaussian symmetric measure with the characteristic function $e^{-t^{2} / 2}$. This leads to the following equation:

$$
\frac{1}{\sqrt{2 \pi}} e^{-x^{2} / 2}=\int_{0}^{\infty} \frac{\Gamma(s+1)}{\sqrt{\pi} \Gamma(s+1 / 2)}\left(1-\frac{x^{2}}{r^{2}}\right)_{+}^{s-1 / 2} \frac{1}{r} f_{s, 2}(r) d r
$$

where $f_{s, 2}$ denotes the density of $\nu_{s, 2}$. It is easy to check that

$$
f_{s, 2}(r)=\frac{1}{2^{s} \Gamma(s+1)} x^{2 s+1} e^{-x^{2} / 2} \mathbf{1}_{(0, \infty)}(x) .
$$

Let $p<2$. If by $\lambda_{p}$ we denote the distribution of the random variable $\sqrt{\Theta}$, where $\Theta$ is the positive $p / 2$-stable random variable with the Laplace transform $\exp \left\{-(2 t)^{p / 2}\right\}$, then $\omega_{s, 1} \circ \nu_{s, 2} \circ \lambda_{p}=N(0,1) \circ \lambda_{p}$ is symmetric $p$-stable since its characteristic function is of the form

$$
\int_{0}^{\infty} e^{-t^{2} u / 2} \lambda_{p}(d u)=\exp \left\{-|t|^{p}\right\}
$$

We know that

$$
|t|^{p}=\int_{\mathbb{R}}(1-\cos (t x)) \frac{c_{p}}{|x|^{p+1}} d x .
$$

It is easy to show that there exists a suitable constant $K$ such that

$$
\frac{c_{p}}{|x|^{p+1}}=\int_{0}^{\infty} \frac{\Gamma(s+1)}{\sqrt{\pi} \Gamma(s+1 / 2)}\left(1-\frac{x^{2}}{r^{2}}\right)_{+}^{s-1 / 2} \frac{1}{r} \frac{K}{r^{p+1}} d r .
$$

This means that the spectral measure for $p$-stable distribution can be obtained as a mixture of the measure $\omega_{s, 1}$ in the following way:

$$
\begin{aligned}
|t|^{p} & =\int_{\mathbb{R}}(1-\cos (t x)) \int_{0}^{\infty} \frac{\Gamma(s+1)}{\sqrt{\pi} \Gamma(s+1 / 2)}\left(1-\frac{x^{2}}{r^{2}}\right)_{+}^{s-1 / 2} \frac{1}{r} \frac{K}{r^{p+1}} d r d x \\
& =\int_{0}^{\infty} \int_{\mathbb{R}}(1-\cos (t z r)) \omega_{s, 1}(d z) \frac{K}{r^{p+1}} d r \\
& =\int_{0}^{\infty}\left(1-\widehat{\omega_{s, 1}}(t z r)\right) \frac{K}{r^{p+1}} d r .
\end{aligned}
$$

6. $\mu$-weak Lévy measure for $\mu$-weakly infinitely divisible distribution. In this section we will use the construction of the Lévy measure for infinitely divisible distribution given in the monograph of K. Sato [17, section 8] in order to show that $\mu$-infinitely divisible mixture of weakly stable measure is also a mixture of this measure. Till now this result was known only for a stable measure $\mu=\gamma_{\alpha}$ and some restricted family of mixtures (see [13]). 
First we define a set $\mathscr{C}$ of bounded measurable functions from $\mathbb{R}$ to $\mathbb{R}$ satisfying

$$
\begin{aligned}
& c(x)=1+o(|x|) \text { as }|x| \rightarrow 0, \\
& c(x)=O(1 /|x|) \text { as }|x| \rightarrow \infty .
\end{aligned}
$$

The functions $c \in \mathscr{C}$ will replace the function $\mathbf{1}_{B}$, for $B=\{x \in \mathbb{R}:|x| \leqslant 1\}$, which appeared in the classical Lévy-Kchintchine representation for the characteristic function of infinitely divisible distribution. This replacement allows us to use weak convergence technics for Lévy spectral measures because of continuity of functions $c \in C$. K. Sato (see [17]) showed that the Lévy measure can be obtained as a weak limit (in somewhat restricted sense) of a sequence of measures defined by convolution powers of the considered infinitely divisible distribution. Thus we obtain $\mu$-weak Lévy measure by the limit of generalized convolution powers of $\mu$-infinitely divisible distribution $\mu$ mixed with respect to this distribution.

Here are examples of functions $c \in \mathscr{C}$ sometimes used:

$$
c(x)=1 /\left(1+|x|^{2}\right), \quad c(x)=\mathbf{1}_{B}(x)+\mathbf{1}_{1<|x| \leqslant 2}(x)(2-|x|) .
$$

Let us write $f \in C_{\#}$ if $f$ is a bounded continuous function from $\mathbb{R}$ to $\mathbb{R}$ vanishing on a neighborhood of zero.

Lemma 4. Assume that $\mu$ is a symmetric weakly stable measure on $\mathbb{R}$ and $\lambda$ is a $\mu$-weakly infinitely divisible measure. Then for any sequence $t_{n} \searrow 0$ we have

$$
\int_{\mathbb{R}} e^{i t x}(\mu \circ \lambda)(d x)=\lim _{n \rightarrow \infty} \exp \left\{-\int_{\mathbb{R}}(1-\widehat{\mu}(t s)) t_{n}^{-1} \lambda^{t_{n}}(d s)\right\} .
$$

Moreover, if $M$ is the Lévy spectral measure for $\mu \circ \lambda$, then for every $f \in C_{\#}$

$$
\lim _{n \rightarrow \infty} \int_{\mathbb{R} \backslash\{0\}} f(x) t_{n}^{-1}\left(\mu \circ \lambda^{t_{n}}\right)(d x)=\int_{\mathbb{R} \backslash\{0\}} f(x) M(d x) .
$$

P r o o f. Since $\lambda$ is $\mu$-weakly infinitely divisible, we have that $\eta=\mu \circ \lambda$ is infinitely divisible in a usual sense and according to the proof of Theorem $8(\mathrm{i})$ in [17] we have $\operatorname{Exp}\left(t_{n}^{-1} \eta^{* t_{n}}\right) \rightarrow \eta \quad$ as $n \rightarrow \infty$. Now it is easy to notice that

$$
t_{n}^{-1}(\mu \circ \lambda)^{* t_{n}}=\mu \circ\left(t_{n}^{-1} \lambda^{t_{n}}\right) .
$$

The last statement of the lemma is a simple implication of Corollary 8.9 in $[17]$

Lemma 5. For each $a>0$

$$
\limsup _{n \rightarrow \infty} t_{n}^{-1} \lambda^{t_{n}}\left([-a, a]^{c}\right)<\infty
$$


P r o o f. Since $\mu$ is a nontrivial weakly stable measure, it contains no atoms and there exists $p>0$ such that $\mu\left([-p, p]^{c}\right)=1 / 2$. Infinite divisibility of $\mu \circ \lambda$ implies that for every $a>0$

$$
\limsup _{n \rightarrow \infty} t_{n}^{-1}(\mu \circ \lambda)^{* t_{n}}\left([-a p, a p]^{c}\right)<\infty .
$$

Now we have

$$
\begin{aligned}
t_{n}^{-1}(\mu \circ \lambda)^{* t_{n}}\left([-a p, a p]^{c}\right) & =t_{n}^{-1} \int_{\{x:|x|>a p\}}(\mu \circ \lambda)^{* t_{n}}(d x) \\
& =t_{n}^{-1} \int_{\{x:|x s|>a p\}} \mu(d x) \lambda^{t_{n}}(d s) \\
& \geqslant \int_{\{x:|x|>p\}} \mu(d x) t_{n}^{-1} \int_{\{s:|s|>a\}} \lambda^{t_{n}}(d s) \\
& =\frac{1}{2} t_{n}^{-1} \lambda^{t_{n}}\left([-a, a]^{c}\right),
\end{aligned}
$$

which was to be shown.

Lemma 6. The sequence of measures $\left\{t_{n}^{-1} \lambda^{t_{n}}\right\}$ is tight outside every open neighborhood of zero.

P r o o f. Since $\left\{t_{n}^{-1}(\mu \circ \lambda)^{* t_{n}}\right\}$ is tight outside every open neighborhood of zero, for every $\varepsilon>0$ there exists $k>0$ such that for every $n \in \mathbb{N}$

$$
t_{n}^{-1}(\mu \circ \lambda)^{* t_{n}}\left([-k p, k p]^{c}\right)<\frac{\varepsilon}{2} .
$$

Similarly as before we can show that

$$
t_{n}^{-1}(\mu \circ \lambda)^{* t_{n}}\left([-k p, k p]^{c}\right) \geqslant \frac{1}{2} t_{n}^{-1} \lambda^{t_{n}}\left([-k, k]^{c}\right),
$$

thus

$$
t_{n}^{-1} \lambda^{t_{n}}\left([-k, k]^{c}\right)<\varepsilon .
$$

Lemma 7. There exists a $\sigma$-finite measure $\nu$ on $\mathbb{R}$ and a subsequence $\left(n_{j}\right)$ such that the sequence $t_{n_{j}}^{-1} \lambda^{t_{n_{j}}}$ converges weakly to $\nu$ outside every open neighborhood of zero.

P r o o f. To show this we choose $a_{m}, m \in \mathbb{N}$ such that $a_{m} \searrow 0$ and let $B_{m}=\left[-a_{m}, a_{m}\right]^{c}$. Since $t_{n}^{-1} \lambda^{t_{n}}$ is tight on $B_{1}$ there exists a measure $\nu_{1}$ on $B_{1}$ and subsequence $t_{n_{k}}^{-1} \lambda^{t_{n_{k}}}$ such that $t_{n_{k}}^{-1} \lambda^{t_{n_{k}}}$ converges weakly to $\nu_{1}$ on $B_{1}$. The sequence $t_{n_{k}}^{-1} \lambda^{t_{n_{k}}}$ is tight on $B_{2}$ as a subsequence of $t_{n}^{-1} \lambda^{t_{n}}$, thus there exists a measure $\nu_{2}$ on $B_{2}$ and a subsequence $t_{n_{k_{i}}}^{-1} \lambda^{t_{n_{k_{i}}}}$ such that $t_{n_{k_{i}}}^{-1} \lambda^{t_{n_{k_{i}}}}$ converges weakly to $\nu_{2}$ on $B_{2}$. Since $B_{2} \supset B_{1}$, we have that $\nu_{2}(A)=\nu_{1}(A)$ for every Borel set $A \subset B_{1}$. Repeating this procedure we can construct a sequence of finite measures $\nu_{m}$ supported in $B_{m}, m \in \mathbb{N}$, such that $\nu_{k}(A)=\nu_{j}(A)$ for $k>j$ and every Borel set $A \subset B_{j}$. Using the diagonal method we 
can also choose a subsequence $t_{n_{j}}^{-1} \lambda^{t_{n_{j}}}$ converging to $\nu_{m}$ on $B_{m}$ for every $m \in \mathbb{N}$. Consequently, the sequence $t_{n_{j}}^{-1} \lambda^{t_{n_{j}}}$ converges weakly outside every open neighborhood of zero to the $\sigma$-finite measure $\nu$ on $\mathbb{R} \backslash\{0\}$ defined by

$$
\nu(A)=\lim _{m \rightarrow \infty} \nu_{m}(A) \text {. }
$$

Lemma 8. The Lévy spectral measure $M$ for the measure $\mu \circ \lambda$ is a scale mixture of the measure $\mu$ and $M=\mu \circ \nu$.

P r o o f. Let $\mathbb{R}_{0}=\mathbb{R} \backslash\{0\}$. We know that for every $f \in C_{\#}$

$$
\lim _{j \rightarrow \infty} \int_{\mathbb{R}_{0}} f(x) t_{n_{j}}^{-1}\left(\mu \circ \lambda^{t_{n_{j}}}\right)(d x)=\int_{\mathbb{R}_{0}} f(x) M(d x) .
$$

On the other hand,

$$
\begin{array}{rl}
\int_{\mathbb{R}_{0}} & f(x) t_{n_{j}}^{-1}\left(\mu \circ \lambda^{t_{n_{j}}}\right)(d x)=\int_{\mathbb{R}_{0}} \int_{\mathbb{R}} f(x s) t_{n_{j}}^{-1} \lambda^{t_{n_{j}}}(d s) \mu(d x) \\
= & \int_{\mathbb{R}_{0}} \int_{B_{m}} f(x s) t_{n_{j}}^{-1} \lambda^{t_{n_{j}}}(d s) \mu(d x) \rightarrow \int_{\mathbb{R}_{0}} \int_{B_{m}} f(x s) \nu_{m}(d s) \mu(d x) \\
= & \int_{\mathbb{R}_{0}} \int_{\mathbb{R}} f(x s) \nu(d s) \mu(d x),
\end{array}
$$

where the third formulation in these calculaions follows from the fact that for $f \in C_{\#}$ we have $x \neq 0$ in the area of integration and for every fixed $x \neq 0$ there exists $m \in N$ such that $f(x s)=f(x s) \mathbf{1}_{B_{m}}(s)$ Consequently, for every $f \in C_{\#}$ we have

$$
\int_{\mathbb{R}_{0}} f(x) M(d x)=\int_{\mathbb{R}_{0}} \int_{\mathbb{R}} f(x s) \nu(d s) \mu(d x),
$$

which was to be shown.

Theorem 2. Assume that $\mu$ is a nontrivial symmetric weakly stable measure on $\mathbb{R}$ such that $\varkappa(\mu)>0$. A measure $\lambda$ is $\mu$-weakly infinitely divisible if and only if there exist $A \geqslant 0$ and a $\sigma$-finite measure $\nu$ on $\mathbb{R}_{0}$ such that $\nu\left([-a, a]^{c}\right)<\infty$ for each $a>0$,

$$
\int_{0}^{\infty} \mu\left([-s, s]^{c}\right) \nu(d s)<\infty
$$

and

$$
\int_{\mathbb{R}} e^{i t x}(\mu \circ \lambda)(d x)=\exp \left\{-A|t|^{\varkappa(\mu)}-\int_{\mathbb{R}_{0}}(1-\widehat{\mu}(t s)) \nu(d s)\right\} .
$$

P r o o f. Since $M=\mu \circ \nu$ is the Lévy measure for the infinitely divisible measure $\mu \circ \lambda$, we have $M\left([-1,1]^{c}\right)<\infty$ and $\int\left(x^{2} \wedge 1\right) M(d x)<\infty$. Now we see that

$$
\begin{aligned}
\mu \circ \nu\left([-1,1]^{c}\right) & =\int_{\mathbb{R}} \int_{|x|>1 /|s|} \mu(d x) \nu(d s) \\
& =\int_{\mathbb{R}}\left(1-F\left(|s|^{-1}\right)+F\left(-|s|^{-1}\right)\right) \nu(d s)<\infty
\end{aligned}
$$


where $F$ is a cumulative distribution function for the measure $\mu$. Consider now

$$
\begin{aligned}
\int\left(x^{2} \wedge 1\right) M(d x) & =\iint\left[x^{2} s^{2} \mathbf{1}(|x s|<1)+\mathbf{1}(|x s|>1)\right] \mu(d x) \nu(d s) \\
& =\int_{\mathbb{R}} s^{2} \int_{|x|<1 /|s|} x^{2} \mu(d x) \nu(d s)+\mu \circ \nu\left([-1,1]^{c}\right) .
\end{aligned}
$$

Integrating by parts the inner integral in the first component of the last sum we obtain

$$
\begin{aligned}
& \int_{|x|<1 /|s|} x^{2} \mu(d x)=\left.x^{2} F(x)\right|_{-|s|^{-1}} ^{|s|^{-1}}-2 \int_{0}^{|s|^{-1}} x F(x) d x-2 \int_{|s|^{-1}}^{0} x F(x) d x \\
& =-s^{-2}\left(1-F\left(|s|^{-1}\right)+F\left(-|s|^{-1}\right)\right)+2 \int_{0}^{|s|^{-1}} x(1-F(x)+F(-x)) d x .
\end{aligned}
$$

Finally we obtain

$$
\int\left(x^{2} \wedge 1\right) M(d x)=\int_{\mathbb{R}} 2 s^{2} \int_{0}^{|s|^{-1}} x(1-F(x)+F(-x)) d x \nu(d s)<\infty .
$$

C a s e 1. Assume that $\varkappa(\mu)=2$. According to Corollary 1 we have $\int x^{2} \mu(d x)=\sigma^{2}<\infty$. For every $\varepsilon>0$ we can choose $s_{0}$ small enough to have

$$
\int_{0}^{|s|^{-1}} x(1-F(x)+F(-x)) d x>\sigma^{2}-\varepsilon
$$

for each $s<s_{0}$. Then

$$
\begin{aligned}
\infty & >\int_{\mathbb{R}} 2 s^{2} \int_{0}^{|s|^{-1}} x(1-F(x)+F(-x)) d x \nu(d s) \\
& >\int_{|s|<s_{0}} 2 s^{2} \int_{0}^{|s|^{-1}} x(1-F(x)+F(-x)) d x \nu(d s) \\
& \geqslant \int_{|s|<s_{0}} 2 s^{2}\left(\sigma^{2}-\varepsilon\right) \nu(d s)=2\left(\sigma^{2}-\varepsilon\right) \int_{|s|<s_{0}}|s|^{\varkappa(\mu)} \nu(d s) .
\end{aligned}
$$

C a s e 2. If $\varkappa(\mu)<2$, then, with the notation $G(x)=1-F(x)+$ $F(-x)$, we have

$$
\frac{G(x s)}{G(s)} \geqslant|x|^{-\varkappa(\mu)}=\lim _{s \rightarrow \infty} \frac{G(x s)}{G(s)} .
$$

Now we can write

$$
\begin{aligned}
\infty & >\int\left(x^{2} \wedge 1\right) M(d x)=\int_{\mathbb{R}} 2 s^{2} \int_{0}^{|s|^{-1}} x G(x) d x \nu(d s) \\
& =\int_{\mathbb{R}} 2 G\left(|s|^{-1}\right) \int_{0}^{1} x \frac{G\left(x|s|^{-1}\right)}{G\left(|s|^{-1}\right)} d x \nu(d s) \\
& \geqslant \int_{0}^{\infty} 4 G\left(s^{-1}\right) \int_{0}^{1} x^{1-\varkappa(\mu)} d x \nu(d s)=\frac{4}{2-\varkappa(\mu)} \int_{0}^{\infty} 2 G\left(s^{-1}\right) \nu(d s) .
\end{aligned}
$$


By Lemma 4 the logarithm of the characteristic function of $\mu \circ \lambda$ is equal to the limit as $n \rightarrow \infty$ of the following integral:

$$
\begin{aligned}
\int_{\mathbb{R}}(1 & -\widehat{\mu}(t s)) t_{n}^{-1} \lambda^{t_{n}}(d x) \\
& =\int_{[-\varepsilon, \varepsilon]}(1-\widehat{\mu}(t s)) t_{n}^{-1} \lambda^{t_{n}}(d x)+\int_{[-\varepsilon, \varepsilon]^{c}}(1-\widehat{\mu}(t s)) t_{n}^{-1} \lambda^{t_{n}}(d x)
\end{aligned}
$$

for arbitrary $\varepsilon>0$. Since $t_{n}^{-1} \lambda^{t_{n}}$ converges weakly outside every neighborhood of zero to the measure $\nu$ and $\int_{-1}^{1}|s|^{\varkappa(\mu)} \nu(d s)<\infty$, we have

$$
\lim _{\varepsilon \searrow 0} \lim _{n \rightarrow \infty} \int_{[-\varepsilon, \varepsilon]^{c}}(1-\widehat{\mu}(t s)) t_{n}^{-1} \lambda^{t_{n}}(d x)=\int_{\mathbb{R} \backslash\{0\}}(1-\widehat{\mu}(t s)) \nu(d s) .
$$

This implies that the limit

$$
\lim _{\varepsilon \searrow 0} \lim _{n \rightarrow \infty} \int_{[-\varepsilon, \varepsilon]^{c}}(1-\widehat{\mu}(t s)) t_{n}^{-1} \lambda^{t_{n}}(d s)
$$

exists and it is finite. Since

$$
\lim _{s \rightarrow 0} \frac{1-\widehat{\mu}(t s)}{1-\widehat{\mu}(s)}=|t|^{\varkappa(\mu)},
$$

it follows that

$$
\begin{aligned}
& \lim _{\varepsilon \searrow 0} \limsup _{n \rightarrow \infty} \int_{[-\varepsilon, \varepsilon]} \frac{1-\widehat{\mu}(t s)}{1-\widehat{\mu}(s)}(1-\widehat{\mu}(s)) t_{n}^{-1} \lambda^{t_{n}}(d s) \\
& \quad=|t|^{\varkappa(\mu)} \lim _{\varepsilon \searrow 0} \limsup _{n \rightarrow \infty} \int_{[-\varepsilon, \varepsilon]}(1-\widehat{\mu}(s)) t_{n}^{-1} \lambda^{t_{n}}(d s)=A|t|^{\varkappa(\mu)}
\end{aligned}
$$

where

$$
A=\lim _{\varepsilon \searrow 0} \limsup _{n \rightarrow \infty} \int_{[-\varepsilon, \varepsilon]}(1-\widehat{\mu}(s)) t_{n}^{-1} \lambda^{t_{n}}(d s) .
$$

Theorem 3. Let $\mu$ be a nontrivial symmetric weakly stable measure on $\mathbb{R}$ such that $\varkappa(\mu)>0$. If the measure $\nu$ on $\mathbb{R}_{0}$ is such that $\nu\left([-a, a]^{c}\right)<\infty$ for each $a>0$ and the condition (4) is satisfied, then for each $A \geqslant 0$ there exists a $\mu$-weakly infinitely divisible measure $\lambda$ such that the right-hand side of (5) is the characteristic function of the measure $\mu \circ \lambda$.

P r o o f. Notice first that if $\varkappa=\varkappa(\mu)>0$, then by Theorem 1 for each $0<p<\varkappa$ there exists a measure $\lambda_{p}$ such that $\mu \circ \lambda_{p}=\gamma_{p}$, where $\widehat{\gamma_{p}}(t)=\exp \left\{-|t|^{p}\right\}$. Choose $p_{n} \nearrow \varkappa$. Since $\exp \left\{-|t|^{p_{n}}\right\} \rightarrow \exp \left\{-|t|^{\varkappa}\right\}$, if $n \rightarrow \infty$, then the family of measures $\left\{\mu \circ \lambda_{p_{n}}: n \in \mathbb{N}\right\}$ is tight. By Lemma 2 in [12] the family $\mathscr{A}=\left\{\lambda_{p_{n}}: n \in \mathbb{N}\right\}$ is also tight. Taking $\lambda_{0}$ for any accumulation point of $\mathscr{A}$ we see that $\mu \circ \lambda_{0}=\gamma_{\varkappa}$. Consequently, for $\nu \equiv 0$ it is enough to take $\lambda=T_{A^{1 / \varkappa}} \lambda_{0}$. 
Now without lost of generality we can assume that $A=0$. We need to show that for every measure $\nu$ satisfying our assumptions there exists a $\mu$-weakly infinitely divisible measure $\lambda$ such that

$$
\int_{\mathbb{R}} e^{i t x}(\mu \circ \lambda)(d x)=\exp \left\{-\int_{\mathbb{R}_{0}}(1-\widehat{\mu}(t s)) \nu(d s)\right\} .
$$

To see this we define a sequence of measures $m_{n}, n \in \mathbb{N}$, by the formula $m_{n}(B)=\nu(B \cap[-1 / n, 1 / n])$ for every Borel set $B$ in $\mathbb{R}$. Then $\lambda_{n}=\operatorname{Exp}_{\otimes_{\mu}}\left\{m_{n}\right\}$ is a well defined probability measure and

$$
\begin{aligned}
\int_{\mathbb{R}} e^{i t x}\left(\mu \circ \lambda_{n}\right)(d x) & =\exp \left\{-\int_{\mathbb{R}_{0}}(1-\widehat{\mu}(t s)) m_{n}(d s)\right\} \\
& \stackrel{n \rightarrow \infty}{\longrightarrow} \exp \left\{-\int_{\mathbb{R}_{0}}(1-\widehat{\mu}(t s)) \nu(d s)\right\} .
\end{aligned}
$$

This means that the set $\left\{\mu \circ \lambda_{n}: n \in \mathbb{N}\right\}$ is tight, thus using again Lemma 2 in [12], we have that $\mathscr{A}_{1}=\left\{\lambda_{n}: n \in \mathbb{N}\right\}$ and we can take for $\lambda$ any accumulation point of $\mathscr{A}_{1}$.

The $\mu$-weak infinite divisibility of $\lambda$ follows now by noticing that if $\nu$ is finite outside every open neighborhood of zero and the condition (4) is satisfied for $\nu$, then for every $n \in \mathbb{N}$ the measure $(1 / n) \nu$ has the same properties.

R e m a r k s. (1) From Lemma 4 it follows that every $\mu$-weakly infinitely divisible distribution $\lambda$ is a weak limit of a sequence of $\otimes_{\mu}$-compound Poisson distributions: $\operatorname{Exp}_{\otimes_{\mu}}\left\{t_{n}^{-1} \lambda^{t_{n}}\right\} \rightarrow \lambda$.

(2) If $\mu=\gamma_{\alpha}$ is a symmetric $\alpha$-stable distribution, then $1-F(x)=$ $\gamma_{\alpha}(x, \infty) \sim c_{\alpha} x^{-\alpha}$. Since the $\gamma_{\alpha}$-weak Lévy measure $\nu$ for a $\gamma_{\alpha}$-weakly infinitely divisible distribution $\lambda$ is finite outside every open neighborhood of zero, thus from Theorem 2 we obtain that $\int_{-\varepsilon}^{\varepsilon} s^{\alpha} \nu(d s)<\infty$ for any $\varepsilon>0$.

(3) Let $\omega_{s, 1}$ be a one-dimensional marginal distribution of the uniform distribution $\omega_{s}$ on the unit sphere in $\mathbb{R}^{2(s+1)}$ for some $s>-1 / 2$ in the sense considered by Kingman in [7], i.e., $\omega_{s, 1}$ has the density function given by

$$
\omega_{s, 1}(d x)=\frac{s !}{\sqrt{\pi}(s-1 / 2) !}\left(1-x^{2}\right)_{+}^{s-1 / 2} d x .
$$

We see that every moment of the measure $\mu=\omega_{s, 1}$ exists, thus $\varkappa\left(\omega_{s, 1}\right)=2$. Theorem 1 states that the weak spectral measure $\nu$ for the $\omega_{s, 1}$-infinitely divisible measure $\lambda$ has the property $\int_{-1}^{1} y^{2} \nu(d y)<\infty$ as in the classical situation. Similar property we would have if we had other than $\omega_{s}$ weakly stable distribution with compact support.

Acknowledgement. This paper was written while the second author was a visiting professor of Delft Institute of Applied Mathematics, Delft University of Technology. 


\section{REFERENCES}

1. Cambanis S., Keener R., Simons G. On $\alpha$-symmetric distributions. - J. Multivariate Anal., 1983, v. 13, p. 213-233.

2. Dudley R. M. Real Analysis and Probability. Belmont, Ca.: Wadsworth\& Brooks/Cole Advanced Books\& Software, Wadsworth Inc., 1989.

3. Feller $W$. An Introduction to Probability Theory and its Applications, vol. 2. New York: John Wiley, 1966.

4. Jasiulis B. H. Limit property for regular and weak generalized convolution. - J. Theoret. Probab., 2010, v. 23, № 1, p. 315-327.

5. Jasiulis-Goldyn B. H., Misiewicz J. K. On the uniqueness of the Kendall generalized convolution. - J. Theoret. Probab., 2011, v. 24, № 3, p. 746-755.

6. Kelker D. Infinite divisibility and variance mixtures of the normal distribution. Ann. Math. Statist., 1971, v. 42, № 2, p. 802-808.

7. Kingman J.F.C. Random walks with spherical symmetry. - Acta Math., 1963, v. 109, № 1, p. 11-53.

8. Kucharczak J., Urbanik K. Quasi-stable functions. - Bull. Polon. Acad. Sci. Ser. Sci. Math. Astronom. Phys., 1974, v. 22, № 3, p. 263-268.

9. Kucharczak J., Urbanik K. Transformations preserving weak stability. - Bull. Polon. Acad. Sci. Ser. Sci. Math. Astronom. Phys., 1986, v. 34, № 7, 8, p. 475-486.

10. Lukács E. Characteristic Functions. London: Griffin, 1960.

11. Mazurkiewicz $G$. On the infinite divisibility of scale mixtures of symmetric $\alpha$-stable distributions, $\alpha \in(0,1]$. — Banach Center Publ., 2010, v. 90, p. 79-82.

12. Misiewicz J. K., Oleszkiewicz K., Urbanik K. Classes of measures closed under mixing and convolution. Weak stability. - Studia Math., 2005, v. 167, № 3, p. 195-213.

13. Misiewicz J. K. Infinite divisibility of substable processes. II. Logarithm of probability measure. - J. Math. Sci., 1996, v. 81, № 5, p. 2970-2979.

14. Misiewicz J. K. Weak stability and generalized weak convolution for random vectors and stochastic processes. - IMS Lecture Notes Monog. Ser., 2006, v. 48, p. 109-118.

15. Misiewicz J. K., Mazurkiewicz G. On (c,p)-pseudostable random variables. - J. Theoret. Probab., 2005, v. 18, № 4, p. 837-852.

16. Oleszkiewicz $K$. On $p$-pseudostable random variables, Rosenthal spaces and $\ell_{p}^{n}$ ball slicing. - Lecture Notes in Math., 1807. Berlin: Springer, 2003, p. 188-210.

17. Sato Ken-iti Lévy Processes and Infinitely Divisible Distributions. Cambridge: Cambridge Univ. Press, 2004.

18. Schoenberg I. J. Metric spaces and completely monotonic functions. - Ann. Math., 1938 , v. 38 , p. $811-841$.

19. Urbanik $K$. Remarks on $\mathscr{B}$-stable probability distributions. - Bull. Polon. Sci. Ser. Sci. Math. Astronom. Phys., 1976, v. 24, № 9, p. 783-787.

20. Urbanik K. Generalized convolutions. - Studia Math., 1964, v. 23, p. 217-245.

21. Urbanik K. Generalized Convolutions II. - Studia Math., 1973, v. 45, p. 57-70.

22. Urbanik K. Generalized Convolutions III. - Studia Math., 1984, v. 80, p. 167-189.

23. Urbanik K. Generalized Convolutions IV. — Studia Math., 1986, v. 83, p. 57-95.

24. Urbanik K. Quasi-regular generalized convolutions. — Colloquium Math., 1988, v. 55, № 1 , p. 147-162.

25. Vol'kovich V. On symmetric stochastic convolutions. - J. Theoret. Probab., 1992, v. 5, № 3, p. 417-430.

26. Волькович В. О бесконечно разложимых мерах в алгебрах со случайной сверткой. Проблемы устойчивости стохастических моделей. М.: ВНИИСИ, 1985, с. 15-24.

27. Волькович В. Многомерные В-устойчивые распределения и некоторые обобщенные свертки. Проблемы устойчивости стохастических моделей. М.: ВНИИСИ, 1984 , c. $40-53$. 